\title{
DỨBin
}

Technological University Dublin

ARROW@TU Dublin

Conference Papers

Healthcare Management

2018

\section{A multi-method scheduling framework for medical staff}

Wael Rashwan

Technological University Dublin, wael.rashwan@tudublin.ie

John Fowler

Arizona State University at the Tempe Campus

Amr Arisha

Technological University Dublin, amr.arisha@tudublin.ie

Follow this and additional works at: https://arrow.tudublin.ie/healthcarcon

Part of the Business Administration, Management, and Operations Commons, Health and Medical Administration Commons, and the Technology and Innovation Commons

\section{Recommended Citation}

W. Rashwan, J. Fowler and A. Arisha, "A MULTI-METHOD SCHEDULING FRAMEWORK FOR MEDICAL STAFF," 2018 Winter Simulation Conference (WSC), 2018, pp. 1464-1475, doi: 10.1109/

WSC.2018.8632247.

This Conference Paper is brought to you for free and open access by the Healthcare Management at ARROW@TU Dublin. It has been accepted for inclusion in Conference Papers by an authorized administrator of ARROW@TU Dublin. For more information, please contact arrow.admin@tudublin.ie, aisling.coyne@tudublin.ie,gerard.connolly@tudublin.ie.

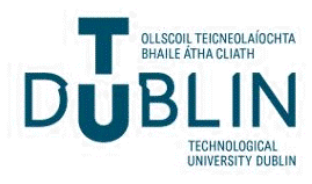




\title{
A MULTI-METHOD SCHEDULING FRAMEWORK FOR MEDICAL STAFF
}

\author{
Wael Rashwan \\ CeADAR, School of Computing \\ Dublin Institute of Technology (DIT) \\ Focas Campus \\ Dublin 8, IRELAND.
}

John Fowler

\author{
W.P. Carey School of Business \\ Arizona State University \\ BA 401 Main Campus \\ Tempe, Arizona 85281, USA.
}

\author{
Amr Arisha \\ 3S Group - College of Business \\ Dublin Institute of Technology (DIT) \\ Aungier Campus \\ Dublin 2, IRELAND.
}

\begin{abstract}
Hospital planning teams are always concerned with optimizing staffing and scheduling decisions in order to improve hospital performance, patient experience, and staff satisfaction. A multi-method approach including data analytics, modeling and simulation, machine learning, and optimization is proposed to provide a framework for smart and applicable solutions for staffing and shift scheduling. Factors regarding patients, staff, and hospitals are considered in the decision. This framework is piloted using the Emergency Department(ED) of a leading university hospital in Dublin. The optimized base staffing patterns and shift schedules actively contributed to solving ED overcrowding problem and reduced the average waiting time for patients by $43 \%$ compared to the current waiting time of discharged patients. The reduction was achieved by optimizing the staffing level and then determining the shift schedule that minimized the understaffing and overstaffing of the personnel need to meet patient demand.
\end{abstract}

\section{INTRODUCTION}

Shortages in hospital staff, in particular, nurses, represent a critical challenge to decision-makers around the world (Zurn et al. 2005). Smith et al. (2011) argued that many industrialized countries are facing a steady depletion of medical staff which will potentially jeopardize healthcare delivery. In the US alone, the shortage of nurses is projected to reach $29 \%$ by 2020 (HRSA 2002). The UK and Ireland have heavily relied on recruiting nurses from overseas to address the shortfall of nursing staff (Buchan et al. 2005).

Several reasons are believed to be contributing to this problem; aging of the professional population, changes in work climate at hospitals, and financial pressures (Goodin 2003; Murray 2002). Reductions in hospital staffing levels cause several problems for management including: discontinuity of care, long waiting times, increasing costs, high workload, and extended time spent organizing cover (Buchan and Seccombe 1995).

Since healthcare services require the physical presence of medical personnel and patients, there is an inevitable sensitivity to demand-capacity gaps. Acknowledging that a service buffer is not a valid option either economically or strategically, the management of healthcare services are forced to find solutions to demand uncertainty, which in turn creates a workload pressure on the hospital staff. This often causes unintended consequences to their health and safety (Portoghese et al. 2014). The process of hospital staffing 


\section{Rashwan, Fowler, and Arisha}

and scheduling has become incredibly complex due to the workload variability. Also, the number and the skill-mix of the required employees frequently change in the very short-term (Norrish and Rundall 2001).

Over the years, economic factors were the driving motive to underpin staffing and scheduling decisions (Brusco and Jacobs 1995). Today, other factors have been considered including workload balance and staff preferences (Van den Bergh et al. 2013). Understaffing carries risks that damage the quality of care and can cause many aspects of hospital performance to (Clark et al. 2015). A trade-off among multiple perspectives, e.g., fairness, cost, and morale should be considered. Several studies have identified an association between staff scheduling and staff morale (Hegney et al. 2006). Ineffective schedules (Dunn et al. 2005), work pressure (Aiken et al. 2013), working long hours (Bae and Fabry 2013; Rogers 2008), and unfair shifts (Dunn et al. 2005; Wilson 2002) are significant factors that lead to a decline in morale. A direct correlation between appropriate staffing size and lower hospital-related mortality is reported in the literature (Kane et al. 2007; Rothberg et al. 2005). Cost increases indirectly due to high staff turnover (Hayes and Bonnet 2010; Jones 2005), absenteeism (Silvestro and Silvestro 2008), and over-staffing. Therefore, optimizing a staff schedule can play a fundamental role in enhancing the performance of healthcare organizations regarding cost and quality of care as well as increasing staff and patient satisfaction.

This project utilized a multi-disciplinary approach to develop an integrated decision support framework that helps hospital/unit managers in staffing and scheduling their staff. The integration of complementary methods seeks to provide a comprehensive solution that addresses the problem and at the same time delivers a practical tool for understanding the complexity of the hospital system.

\section{RELATED WORK}

The models addressing medical staffing and scheduling can be categorized into two categories: single- and multi-stage models. The former class includes studies that examined one stage of the problem while the latter class considers the integration between the different stages. The integration between the staffing and scheduling decisions should accommodate forecast errors in the staffing decisions and also reduce its implications on the scheduling decisions. It can be argued that under/overstaffing occurs regularly in hospitals as a result of disjoint and independent decisions at different planning and scheduling stages (Li et al. 2007). Review of the published articles indicates that most of the staffing and scheduling efforts have used single-stage models, and only a few publications have attempted to combine multiple planning stages (Kim and Mehrotra 2015).

Within the literature regarding workforce planning, several studies have contextualized the staff planning and scheduling decisions in multiple interdependent stages. In 1973, Abernathy et al. (1973) introduced a three-stage staffing framework. The first stage is related to the policy decisions, which includes operating procedures, and staff control processes. Staff planning is covered in the second stage, which is concerned with decisions such as hiring, firing, training, and reallocation. Finally, the third stage considers short-term scheduling of the available staff. In this framework, the actions taken at lower levels are constrained by decisions at higher levels. Similarly, Campbell (1999) presents a three-level decisionmaking framework: planning, scheduling and allocation. The planning phase determines the staff requirements. The scheduling phase assigns off-days, work days and shifts to the individuals. The allocation phase -often referred to rescheduling or re-rostering-deals with the real-time adjustment of personnel to accommodate demand fluctuations and unplanned staff absences. Another major study outlines a sixmodule framework to classify staff scheduling: demand modelling; days-off schedule; shift scheduling; a line of work construction; task assignment; and employees assignment (Ernst et al. 2004). Module one addresses the demand forecast to determine staff requirements per period and per shift distinguishing between three broad demand incidents: task-based, flexible, and shift-based demand. Modules two, three, and four deal with the three types of scheduling problems that appear in the healthcare system namely daysoff allocation, shift scheduling and tour scheduling problems (Morris and Showalter 1983). The days-off allocation problem arises when the staff's work is different from the facility's working week (i.e. continuous system). The shift schedule deals with selecting from a large pool of shifts to satisfy the demand over some planning horizon. Tour scheduling (line of work construction) combines both shift scheduling and days-off 


\section{Rashwan, Fowler, and Arisha}

scheduling to build feasible work patterns over the planning time horizon. Task assignment allocates jobs (e.g. patients) to staff (e.g. nurses).

\section{METHODOLOGY}

The exploratory stage of this research was designed to identify the underlying elements of staffing and scheduling of medical staff before further steps towards the integrated framework took place. The literature review contributed to establishing the theoretical grounds reported; however, given the applied nature of the study, it was crucial to incorporate the practitioners' perspective in the early phases of framework design. This can help in bridging the gap between theoretical studies and practice by exploring management's understanding of staffing and scheduling in the context of Irish hospitals. The exploratory study consisted of $25 \mathrm{semi}$-structured face-to-face interviews in eleven different hospitals in the Irish healthcare acute system to capture and analyze managers' perspectives in practice (Rashwan 2017). This qualitative inquiry provided a sharp lens into the perception of hospital managers in staffing and scheduling medical staff. The exploration stage identified four aspects of staffing and scheduling as shown below.

- Patient aspect represents the demand side, and it is the primary focus of the hospital services. It determines workload volume, acuity, and the need for care that may introduce uncertainty into the system.

- Staff aspect reflects the supply side regarding the number of available hours, staff size and skillmix.

- Organizational aspect focuses on the organization characteristics, managerial factors and operational processes variables that identify the working environment and affect the ability of medical staff team to be efficient and effective.

- The external aspect includes the factors and variables that are outside the organizational environment and may have direct or indirect impacts on determining the hospital staff planning and scheduling. Examples of these factors that may impose extra constraints are state legislation, labor union regulations, staff shortage/supply, economic factors and restructure and reform of the health services.

The exploration stage found that hospital managers rely on simple average-based methods to manage and plan staffing decisions. Variability, uncertainty, processes, constraints, patient flow rules, physical capacities, coordination with other hospital facilities are among the factors that influence the staffing and scheduling decisions. The current practices, therefore, are inadequate to comprehend the dynamics and complexity associated with the hospital system. Therefore, this paper discusses the conceptual development of the proposed integrated framework for medical staff scheduling in the hospital context.

The framework utilizes a hybrid multi-method modeling and simulation (hybrid M\&S) approach which has the potential to comprehend and understand complex systems. The framework development phases are demand for care, understanding dynamics, and optimization (Figure 1). Phase one utilizes data analytics, statistical analysis and machine learning to address and understand patient demand (patient aspect). Phase two combines process modeling, conceptualization and hybrid simulation to capture the dynamics and complexity of the hospital system. Phase 3 is the optimization phase which has two stages: staff optimizer and shift scheduling. Stage one determines the staffing patterns that match patient demand and considers all the main factors that have been incorporated into the simulation model. In the second stage, the shift schedule optimizer determines the shifts that satisfy the staffing needs obtained from stage one with the goal of minimizing under/overstaffing. The simulation model is the performance evaluator for the schedule solutions obtained. The components of the solution framework are introduced and explained in detail below. 


\section{Rashwan, Fowler, and Arisha}
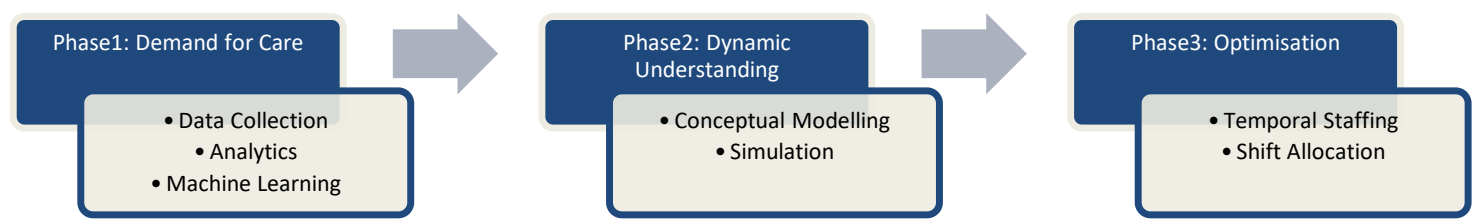

Figure 1: The proposed Framework Phases.

\subsection{Phase 1: Demand for Care}

- Data Collection. Data collection is required across the four aspects of medical staffing and scheduling: patient, staff, organization and external. The framework utilizes various methods of data collection to incorporate multiple perspectives (i.e., qualitative and quantitative). Interviews and observations have a qualitative nature, which is of a high benefit in understanding and modeling of the workflow (processes) in the healthcare facility.

- Analytics Component. Within the proposed framework, incorporating demand patterns, patient profiles, and the related statistical models that determine the important patient factors that require sophisticated techniques. Therefore, the data analytics component helps to assess the multifaceted nature of patient demand considering patient volume, severity mix, and patterns of patient presentations. Descriptive analytics is a key to analyzing the historical input data to capture the insightful information from the data.

- Machine Learning Component for learning decision rules. Staff members make critical sequential decisions during the patients stay such as patient escalation, specialty referral, assigning patient acuity/severity level, or making a disposition decision (admit or discharge). Such decisions have implications on the staff workload and patient care plan. Incorporating machine learning into the simulation model improves the predictability of the model. This framework adopts the Classification and Regression. Tree (CART) algorithm to predict the patient-related decisions within the simulation model.

\subsection{Phase 2: Understanding Dynamics}

Given the systems' path dependencies, time-varying factors (e.g., demand) and the stochastic nature of the systems, system equilibrium is not generally attainable. As a result, modeling the system using steady-state closed-form equations such as queuing networks to account for all underlying factors as previously discussed is not achievable. Alternatively, the underlying system is modeled as a detailed microscopic simulation model that accounts for all problem elements and factors.

- Conceptualization Component. Conceptual modeling is a significant step of model building, and it is potentially the most significant stage in a simulation study. Modeling the underlined business processes requires knowledge from the individuals directly involved in the service delivery. The data collection phase combined interviews, a focus group, and observations from the literature. Since model conceptualization is an iterative process, it requires close interaction with experts and practitioners to obtain holistic insights of the system aspects. Business processes are mapped into a conceptual model using one of the well-developed modeling languages (i.e., flow chart, IDEF2, and state chart diagram) where sub-processes and activities are identified.

- Simulation Components. Discrete-event simulation (DES) offers a better representation of detail complexity and can capture the processes and factors' dynamics (Arisha and Rashwan 2016). The DES model simulates the patient flow in the hospital to capture the resource consumption, particularly staff, over time. It investigates the relationships and interactions among the main factors as identified in the exploratory study and the literature. The factors incorporated are grouped into four categories; for more details refer to (Rashwan 2017). 


\subsection{Phase 3: Optimization}

The third phase has two stages: temporal staffing and shifts staff scheduling.

Temporal Staffing Model uses Discrete Event Simulation (DES) model as the system performance evaluator for the decisions. The staffing optimizer aims to find the (near) optimal staffing patterns $\left(b_{s t}\right)$. This requires solving a simulation optimization problem that considers multiple objectives including minimizing the expected waiting time and patient length of stay (LOS) and balancing staff utilization. The general mathematical formulation of this problem is:

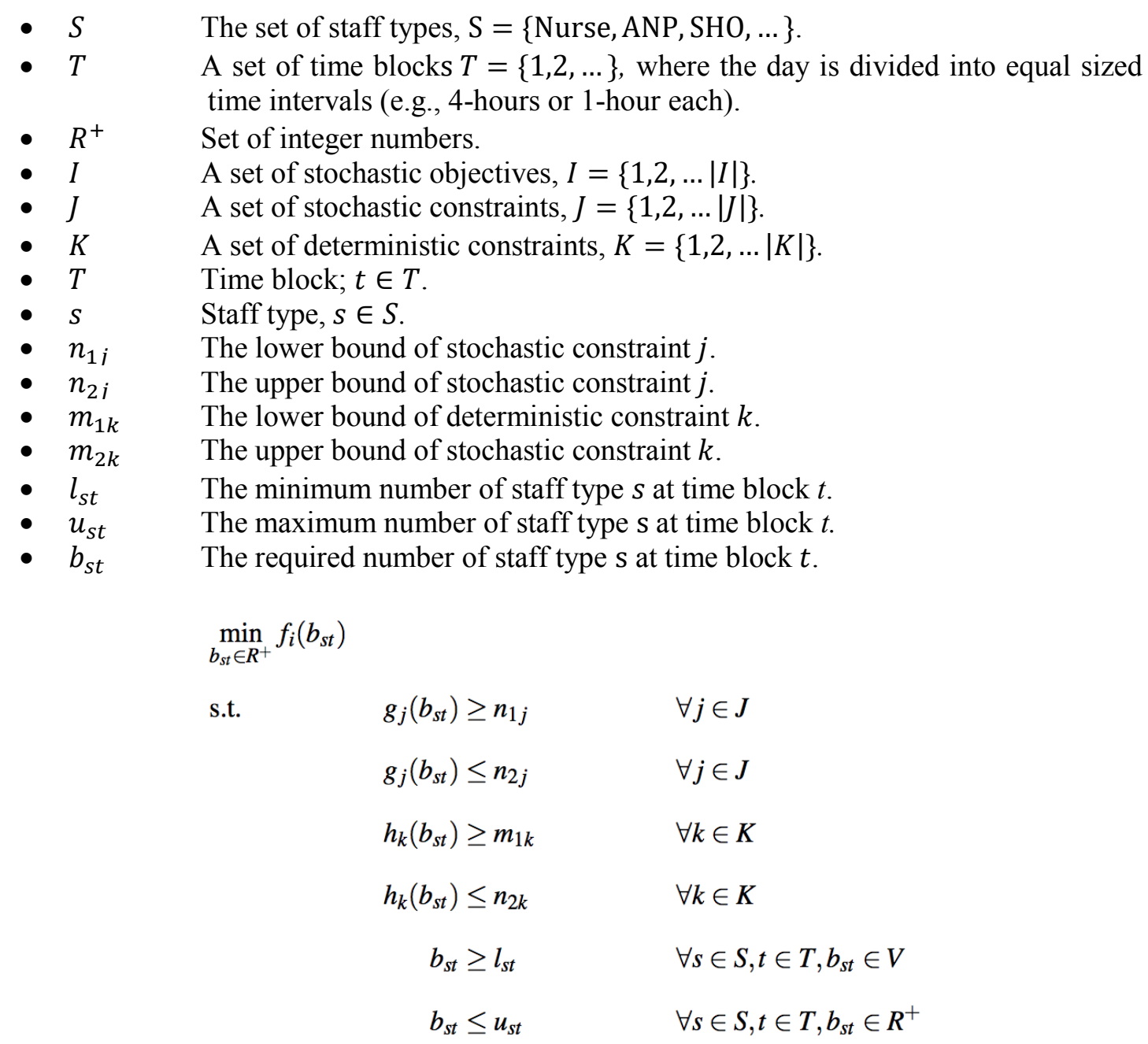

$f_{i}\left(b_{s t}\right)$ is a stochastic performance function $i$, and $g_{j}\left(b_{s t}\right)$ is a stochastic constraint $j$ with lower bound $n_{1 j}$ and upper bound $n_{2 j}$, respectively. Both $f$ and $g$ are evaluated using the simulation. $l_{s t}$ and $u_{s t}$ are lower and upper bounds for the $b_{s t}$, respectively. Other criteria or constraints can be added. This problem is a nonlinear discrete simulation optimization problem which makes the most appropriate optimization method to be metaheuristics or by a state of the art simulation optimization solver. The problem can be reformulated into a single objective problem using the desirability multi-criteria approach (Costa et al. 2011). This approach allocates an overall desirability index to a set of objectives and selects the decision variables settings such that the overall desirability function is maximized.

Flexible Shift Schedule Model feeds optimal temporal staffing requirements, as well as shift patterns into the shift schedule optimizer to determine the shift schedule scheme for each resource type. The decision 


\section{Rashwan, Fowler, and Arisha}

variables in this model are to determine the number of staff resources that start at a specific shift subject to the staffing requirements obtained from the staffing optimizer. This problem is a bi-criteria optimization problem that aims to minimize the understaffing and overstaffing. This formulation is similar to the shift staggered formulation presented by (Sinreich et al. 2012). The list of notation used for formulating the flexible shift schedule model is:

- $\quad M \quad$ A set of different shift lengths. e.g., 12-hour, 11-hour, 8-hour.

- $m$ A shift of length $m, m \in M$

- $b_{s t} \quad$ The required number of staff type s at time block $t$.

- $l_{s t} \quad$ The minimum number of staff type $\mathrm{s}$ at time block $t$.

- $u_{s t}$ The maximum number of staff type s at time block $t$.

- $P_{m s} \quad$ A set of prohibited start times for a shift of length $m$ assigned to staff types, $P_{m s} \subseteq T$.

- $x_{s m}$ The number of staff type s that starts their working shift of length $m$ at time block $k$.

- $y_{s t}$ The number of staff type s allocated to time block $t$.

- $O_{s t}$ The overstaffing of staff type s at time block $t$.

- $U_{s t}$ The understaffing of staff type s at time block $t$.

- $\beta$ Penalty coefficient for being understaffed.

- $\alpha \quad$ Penalty coefficient for being overstaffed.

- $a_{t m k}=\left\{\begin{array}{l}1 \text { if the time block } t \text { is covered in a shift of length } m \text { that starts at } k \text {. } \\ 0 \text { Otherwise }\end{array}\right.$

$$
\begin{aligned}
& \min _{x_{s m k}, O_{s t}, U_{s t} \in R^{+}} \sum_{t} \beta \cdot U_{s t}+\sum_{t} \alpha \cdot O_{s t} \\
& \text { s.t. } \quad y_{s t}=\sum_{k \in P_{m s}} a_{t m k} \cdot x_{s m k} \quad \forall s \in S, t \in T \\
& y_{s t} \geq l_{s t} \quad \forall s \in S, t \in T \\
& y_{s t} \leq u_{s t} \quad \forall s \in S, t \in T \\
& O_{s t}-U_{s t}=y_{s t}-b_{s t} \quad \forall s \in S, t \in T \\
& x_{s m k}, O_{s t}, U_{s t} \geq 0 \quad \forall s \in S, t \in T, k \in K, \text { and } x_{s m k} \in R^{+}
\end{aligned}
$$

The objective function minimizes the total penalized understaffing and overstaffing across all time blocks $t$. The First constraint describes the number of staff of type $s$ that work at time block t. The second and third constraints preserve the minimum and the maximum number of staff of type $s$ that work at time block t, respectively. The fourth constraints calculate the amount of understaffing and overstaffing. This constraint linearizes the model by making $U_{s t}$ and $O_{s t}$ decision variables instead of auxiliary variables. Finally, the last constraint is the non-negativity constraint and integrity constraint. This model uses a mixed linear programming model Mixed Integer Program (MIP). A Branch and Bound (B\&B) algorithm is a candidate to solve the linear relaxation of this problem. Also, the model can be solved for each staff type separately.

This formulation allows the model to select the shifts start times and the number of staff assigned to each to match the fluctuated demand. This adds more flexibility for addressing the shifted demand using different shift lengths and flexible start times than using fixed shifts. 


\section{Rashwan, Fowler, and Arisha}

\section{FRAMEWORK VALIDATION: A CASE STUDY}

The objective of this section is to evaluate the usefulness of the proposed framework as a foundation for addressing the staffing and shift scheduling in a hospital context. A typical case study in a hospital partner is selected to implement the proposed framework in the Emergency Department. The partner hospital has one of the largest Emergency Department nationally. The department has officially, 31 monitored trolley spaces; 5 of these trolley spaces (resuscitation area) are reserved for major trauma and critical care patients. The remaining areas are divided into two zones: Majors Zone with a capacity of 18 trolley spaces, and Minors Zone with eight trolley spaces. The ED also provides two triage rooms. Five distinct areas can be identified: a waiting room for walk-in patients waiting for triage, a diagnostics area (e.g., X-Ray), an ED resuscitation area, an ED major assessment area, and an ED minor assessment area.

In the partner hospital, the staff faces several challenges to maintain a safe, dignified, and high-quality of healthcare service considering the pressures of delivering a range of demanded services to its catchment area. Management struggles to set the adequate staffing levels to meet the sustained requirement to keep the waiting time to acceptable levels. These pressures are significant in the nursing and medical discipline, where both national and international competitive factors have produced inadequate resource levels.

The sample data collected from all anonymous acute patients were gathered retrospectively for one year for patients that presented to the ED between April 1st, 2016 and March 30th, 2017. The data collected for this project utilized both quantitative and qualitative data types. The direct and indirect time per activity and staff schedule were not stored in the IT system. This data was collected from interviews and observations. The qualitative data such as pathways, routing, and conceptual modeling were gathered through observation, interviews, and focus groups. Phase 1 and 2 of the framework were applied to the case. Accordingly, a comprehensive DES simulation model for the ED was constructed using the Anylogic simulation package. The DES model included all the factors mentioned from the patient, staff and organizational aspects. Several CART prediction models were integrated into the DES model in the form of decision-rules. All model inputs were stored in a database attached to the simulation model. The model output is exported to an Excel database for further analysis and validation.

For the verification process, the model logic is scrutinized to ensure that patients follow the correct care path as expected. It was also achieved through checking intermediate output values such as queue lengths and waiting times between processes. The conceptual model was documented and validated by circulating the document among ED senior consultants and senior nursing staff. The final results of the simulation model were validated using six key performance indicators (KPIs). The actual averages were calculated after removing the outliers using the 10th and 90th percentiles as the cut-off. The simulation model was run for $n=20$ independent replications to obtain independent and identically distributed (iid) of KPIs above, with each replicate re-initialized by a different pseudorandom number seed. Smart Staff Allocation - This analysis focused on five types of ED staff: Triage Nurse, Advanced Nurse Practitioner (ANP), ED registered nurse, ED SHO (Senior House Officer) and ED Registrar. The ED staff in the partner hospital are all dedicated to the ED. Nurses work two long shifts 8 AM to 8 PM and 8 PM to 8 AM. The SHO team has six different shifts during the day, and the Registrar staff have five. Similarly, the shift duration of doctors is set at 10 hours. Fixed length shifts with predetermined starts are inflexible to meet the demand patterns that change from one moment to another.

Table 1 summarizes the percentage change between the values of the KPIs calculated from the analysis of the patient records and the output of the simulation model. For each KPI, 95\% confidence intervals based on the t-distribution are estimated. Since a simulation model of a real-world system is only an approximation to the actual system, one should not speak of absolute validity or invalidity of a model, but instead of the degree to which the model reflects the system. The relative differences between the actual system and the simulated model reveal that there are some over-/under-estimations. However, the discrepancies are practically insignificant, and they are in acceptable ranges $( \pm 5 \%)$. Although there are some statistical significance between actual and simulated, the this is deemed to be not practically significant. The model, for all practical purposes, is a reasonably valid representation of the real system. 


\subsection{Smart Staff Allocation}

This analysis focused on five types of ED staff: Triage Nurse, Advanced Nurse Practitioner (ANP), ED registered nurse, ED SHO (Senior House Officer) and ED Registrar. The ED staff in the partner hospital are all dedicated to the ED. Nurses work two long shifts 8 AM to 8 PM and 8 PM to 8 AM. The SHO team has six different shifts during the day, and the Registrar staff have five. Similarly, the shift duration of doctors is set at 10 hours. Fixed length shifts with predetermined starts are inflexible to meet the demand patterns that change from one moment to another.

Table 1: Simulation output vs. actual (one-year simulation).

\begin{tabular}{|c|c|c|c|c|c|c|}
\hline Run & ED PET $^{1}$ & $\begin{array}{l}\text { ED PET } \\
\text { Admitted }^{2}\end{array}$ & $\begin{array}{l}\text { ED PET } \\
\text { Discharged }^{3}\end{array}$ & $\begin{array}{l}\text { ED Admission } \\
\text { Rate }^{4}\end{array}$ & $\%<6 \mathrm{hrs}^{5}$ & $\%<9 \mathrm{hrs}^{6}$ \\
\hline Mean & 530.36 & 776.47 & 353.57 & 0.24 & 0.4949 & 0.6622 \\
\hline Stdev. & 12.27 & 10.95 & 14.71 & 0.002 & 0.0083 & 0.0088 \\
\hline Half CI & 5.74 & 5.12 & 6.89 & 0.001 & 0.0039 & 0.0038 \\
\hline LB & 524.6 & 771.35 & 346.68 & 0.23 & 0.4910 & 0.6581 \\
\hline UB & 536.1 & 781.59 & 360.45 & 0.24 & 0.498854 & 0.66 \\
\hline Actual & 514.0 & 790 & 371.00 & 0.23 & 0.47 & 0.66 \\
\hline$\% \operatorname{diff}$ & $-3 \%$ & $2 \%$ & $5 \%$ & $-1 \%$ & $5.31 \%$ & $0.34 \%$ \\
\hline
\end{tabular}

Management has selected two main criteria that determine the quality of service. The average waiting time for patients and the percent of patients whose LOS is less than six hours are both reflections of the patient experience time. Both performance indicators are aligned with the national strategy that aims to improve the safety and quality of care as stated by of the Emergency Medicine Programme (EMP). From the staff perspective, sustained working close to full utilization to fulfill the excess workload with minimal cost leads to substantial implications and ultimately reduces the productivity and may increase the operating costs. Therefore, an efficient schedule should keep the staff utilization to be less than a threshold.

The three criteria are evaluated using the simulation model, therefore obtaining the (near) optimal staffing patterns requires solving a stochastic multi-objective simulation optimization problem with integer variables. The Desirability approach was used to optimize multiple simultaneous objectives by transforming the estimated response (by simulation) into a unified scale called a desirability index. The desirability functions used and the parameters are obtained from Rashwan (2017). The resultant instance of temporal staffing model is solved using OptQuest solver. The results of the staffing optimizer stage are summarised in Table 2. The staffing optimizer has achieved a significant reduction in required daily staffing hours compared to the current staffing profiles. Nursing and triage hours were reduced by 36 and 14 respectively, while ANP hours increased by 11 hours. Registrar hours increased significantly from 58 hours to 81 hours (23 extra hours are required). This has been compensated by a substantial reduction in the SHO hours (16 hours).

However, the adopted fixed shifts by the department do not help management to benefit from these reductions. For example, nurses are working single length shift (12-hours) with the fixed start time (at 8 AM and PM). Therefore, the shift schedule that satisfies the staffing requirements is the maximum staff required during the first shift, which is ten nurses. Similarly, the second shift will be allocated ten nurses. This simple schedule is inefficient regarding overstaffing and unfair regarding workloads. The flexibility of shifts with variable length and start times enables management to better respond to the demand efficiently and effectively. In stage two of the optimization phase, various shifts with variable starting times were explored to predict their implications on the over/understaffing.

The results obtained from the staffing optimizer were fed into the shift scheduler to determine the optimal shift starts for each staff type. In this experiment, four types of shifts are used 12, 10, 8, and 6 hours for nurses, and three shifts 10,8 , and 6 for doctors. 


\section{Rashwan, Fowler, and Arisha}

Table 2: Results obtained from the staffing optimizer.

\begin{tabular}{|c|c|c|c|c|c|c|c|c|c|c|}
\hline \multirow[b]{2}{*}{ Hour } & \multicolumn{2}{|c|}{ Nurse } & \multicolumn{2}{|c|}{ Triage Nurse } & \multicolumn{2}{|c|}{ ANP } & \multicolumn{2}{|c|}{ SHO } & \multicolumn{2}{|c|}{ Registrar } \\
\hline & As is & Optimised & As is & Optimised & As is & Optimised & As is & Optimised & As is & $\overline{\text { Optimised }}$ \\
\hline 0 & 9 & 7 & 2 & 1 & 1 & 1 & 1 & 1 & 2 & 3 \\
\hline 1 & 9 & 7 & 2 & 1 & 1 & 1 & 1 & 1 & 2 & 3 \\
\hline 2 & 9 & 6 & 2 & 1 & 1 & 1 & 1 & 1 & 2 & 2 \\
\hline 3 & 9 & 5 & 2 & 1 & 1 & 1 & 1 & 1 & 2 & 2 \\
\hline 4 & 9 & 5 & 2 & 1 & 1 & 1 & 1 & 1 & 2 & 2 \\
\hline 5 & 9 & 4 & 2 & 1 & 1 & 1 & 1 & 1 & 2 & 1 \\
\hline 6 & 9 & 4 & 2 & 1 & 1 & 1 & 1 & 1 & 1 & 1 \\
\hline 7 & 9 & 4 & 2 & 1 & 1 & 1 & 1 & 1 & 1 & 2 \\
\hline 8 & 10 & 6 & 2 & 1 & 1 & 1 & 1 & 1 & 1 & 3 \\
\hline 9 & 10 & 9 & 2 & 2 & 1 & 2 & 1 & 2 & 2 & 6 \\
\hline 10 & 10 & 9 & 2 & 2 & 1 & 2 & 3 & 2 & 3 & 4 \\
\hline 11 & 10 & 10 & 2 & 2 & 1 & 2 & 3 & 3 & 3 & 4 \\
\hline 12 & 10 & 11 & 2 & 2 & 1 & 2 & 3 & 3 & 3 & 4 \\
\hline 13 & 10 & 10 & 2 & 2 & 1 & 2 & 3 & 2 & 3 & 5 \\
\hline 14 & 10 & 10 & 2 & 2 & 1 & 2 & 4 & 2 & 4 & 4 \\
\hline 15 & 10 & 10 & 2 & 2 & 1 & 2 & 4 & 2 & 4 & 4 \\
\hline 16 & 10 & 9 & 2 & 2 & 1 & 2 & 5 & 2 & 4 & 4 \\
\hline 17 & 10 & 10 & 2 & 2 & 1 & 2 & 5 & 2 & 3 & 4 \\
\hline 18 & 10 & 10 & 2 & 2 & 1 & 2 & 4 & 2 & 3 & 4 \\
\hline 19 & 10 & 10 & 2 & 1 & 1 & 2 & 4 & 2 & 3 & 4 \\
\hline 20 & 9 & 10 & 2 & 1 & 1 & 1 & 2 & 2 & 2 & 4 \\
\hline 21 & 9 & 9 & 2 & 1 & 1 & 1 & 2 & 2 & 2 & 4 \\
\hline 22 & 9 & 9 & 2 & 1 & 1 & 1 & 2 & 2 & 2 & 4 \\
\hline 23 & 9 & 8 & 2 & 1 & 1 & 1 & 2 & 1 & 2 & 3 \\
\hline Total hours & 228 & 192 & 48 & 34 & 24 & 35 & 56 & 40 & 58 & 81 \\
\hline change & & -36 & & -14 & & 11 & & -16 & & 23 \\
\hline
\end{tabular}

As described in chapter 5, formulation three is applied to obtain the staff shift schedule that minimizes the understaffing and overstaffing for each staff type with more weight given to the understaffing to maintain both quality of care and improve staff satisfaction. The results of the shift scheduler for registered nurse staff is shown in Table 3. Their schedule consists of working the four shifts. The first shift is 12-hour shift that is set as follows: three nurses start at 7 AM and 7 PM while only one nurse at 1 PM, 3 PM, 4 PM and $8 \mathrm{PM}$. The 10-hour shift is registered with three nurses; two at $10 \mathrm{AM}$ and one at $12 \mathrm{PM}$. Only one nurse is planned for 8-hour shift that starts at 9 AM. The last scheduled shift for nurses is the 6-hour shift which is scheduled for three nurses; one nurse starts at 8 AM, 9 AM, and 10 AM.

Table 3: The optimal shift schedule for registered nurse staff.

\begin{tabular}{|c|c|c|c|c|c|c|c|c|c|c|c|c|c|c|c|c|c|c|c|c|c|c|c|c|c|c|}
\hline \multirow{6}{*}{$\overline{\mathbf{Z}}$} & $\mathrm{H}^{5}$ & 0 & 1 & 2 & 3 & 4 & 5 & 6 & 7 & 8 & 9 & 10 & 11 & 12 & 13 & 14 & 15 & 16 & 17 & 18 & 19 & 20 & 21 & 22 & 23 & Sum \\
\hline & $\begin{array}{ll}\mathbf{R}^{6} \\
\end{array}$ & 7 & 7 & 7 & 6 & 5 & 5 & 4 & 4 & 4 & 6 & 9 & 9 & 10 & 11 & 10 & 10 & 10 & 9 & 10 & 10 & 10 & 10 & 9 & 9 & 192 \\
\hline & 12h S & 0 & 0 & 0 & 0 & 0 & 0 & 0 & 3 & 0 & 0 & 0 & 0 & 0 & 1 & 0 & 1 & 1 & 0 & 0 & 3 & 1 & 0 & 0 & 0 & \\
\hline & $10 \mathrm{~h} \mathrm{~S}$ & 0 & 0 & 0 & 0 & 0 & 0 & 0 & 0 & 0 & 0 & 2 & 0 & 1 & 0 & 0 & 0 & 0 & 0 & 0 & 0 & 1 & 0 & 0 & 0 & \\
\hline & $8 \mathrm{~h} \mathrm{~S}$ & 0 & 0 & 0 & 0 & 0 & 0 & 0 & 0 & 0 & 1 & 0 & 0 & 0 & 0 & 0 & 0 & 0 & 0 & 0 & 0 & 0 & 0 & 0 & 0 & \\
\hline & $6 \mathrm{~h} \mathrm{~S}$ & 0 & 0 & 0 & 0 & 0 & 0 & 0 & 0 & 1 & 1 & 1 & 0 & 0 & 0 & 0 & 0 & 0 & 0 & 1 & 0 & 0 & 0 & 0 & 0 & \\
\hline \multirow{4}{*}{$\mathbf{C}^{2}$} & $12 \mathrm{~h} \mathrm{~S}$ & 6 & 6 & 6 & 5 & 4 & 4 & 4 & 4 & 3 & 3 & 3 & 3 & 3 & 4 & 4 & 5 & 6 & 6 & 6 & 6 & 7 & 7 & 7 & 7 & 120 \\
\hline & $10 \mathrm{~h} \mathrm{~S}$ & 1 & 1 & 1 & 1 & 1 & 1 & 0 & 0 & 0 & 0 & 2 & 2 & 3 & 3 & 3 & 3 & 3 & 3 & 3 & 3 & 2 & 2 & 1 & 1 & 40 \\
\hline & $8 \mathrm{~h} \mathrm{~S}$ & 0 & 0 & 0 & 0 & 0 & 0 & 0 & 0 & 0 & 1 & 1 & 1 & 1 & 1 & 1 & 1 & 1 & 0 & 0 & 0 & 0 & 0 & 0 & 0 & 8 \\
\hline & $6 \mathrm{~h} \mathrm{~S}$ & 0 & 0 & 0 & 0 & 0 & 0 & 0 & 0 & 1 & 2 & 3 & 3 & 3 & 3 & 2 & 1 & 0 & 0 & 1 & 1 & 1 & 1 & 1 & 1 & 24 \\
\hline$\overline{\mathbf{U}^{3}}$ & & & & & & & & & & & & & & & & & & & & & & & & & & 0 \\
\hline $\mathbf{O}^{4}$ & & & & & & & & & & & & & & & & & & & & & & & & & & 0 \\
\hline
\end{tabular}

${ }^{1}$ Registered Nurse, ${ }^{2}$ Coverage, ${ }^{3}$ Understaffing, ${ }^{4}$ Overstaffing, ${ }^{5}$ Hour (time block), ${ }^{6}$ Staff Requirements per time block.

The nurse schedule results in 192 nursing hour schedule that covers the demand requirement with zero planned under/overstaffing. The results of staff schedules from this stage are simulated to evaluate the predicted performance after optimizing the staffing patterns and shift schedule (Table 4). As expected, the 


\section{Rashwan, Fowler, and Arisha}

performance has been improved substantially through modifying the staff patterns to match demand. Optimised staffing patterns eliminated $43 \%$ of the patients waiting time which was reflected in the performance measures. The simulation model predicted the PET of all patients had been dropped by $29 \%$, while PET discharge was reduced by 33\%. Optimising staffing patterns to match the demand has limited impact on reducing the PET of admitted patients because most of their waiting is boarding. Percent of patients discharged or admitted in less than six hours improved by $25 \%$, while there was a $13 \%$ improvement in the number of patients admitted or discharged in less than nine hours.

Table 4: Performance comparison.

\begin{tabular}{lcccccc}
\hline \hline & WT & ED PET & ED PET Admitted & ED PET Discharged & \% $<$ 6hrs & \%< 9hrs \\
\cline { 2 - 8 } Current Schedule & 272 & 530.36 & 776.47 & 353.57 & 0.4949 & 0.6622 \\
Optimised Schedule & 155 & 375 & 733 & 250 & 0.618 & 0.747 \\
\hline \hline$\%$ change & $-43 \%$ & $-29 \%$ & $-6 \%$ & $-33 \%$ & $25 \%$ & $13 \%$ \\
\hline \hline
\end{tabular}

\section{CONCLUSION}

Hospital managers strive to utilize new techniques to better address planning issues. However, most of the Irish hospitals still depend on historical data average based methods for their planning. Subject to the constrained healthcare professional supply, optimizing staffing and shift scheduling decisions of the current doctors and nurses are of immense importance, notably, to enable management's response to system dynamics efficiency. This requires systematic and advanced methods to support management with informed decisions regarding staffing and scheduling based on evidence from data. The developed framework provides a comprehensive solution that enables managers to explore and address the dynamics of factors that impact the staffing and scheduling decisions. Also, it provides a tool to enhance current decision making by considering various factors across the central aspect of the problem: patient, staff, and organizational aspects. This allows better-informed decision-making at operational and tactical levels. The optimized base staffing patterns and shift schedules actively contributed to solving the ED overcrowding problem and reduced the average waiting time for patients by $43 \%$ compared to the current waiting time of discharged patients. That was achieved by optimizing the staffing level and then determining the shift schedule that minimized the understaffing and overstaffing of the personnel needed to meet patient demand. Based on explored experiments from the research, management should utilize flexible shifts to develop effective and efficient staff schedules. Optimal staffing levels are a necessary step that enables hospital managers to align their staff patterns to meet patient needs, but it is not sufficient. Staff schedules based on fixed shifts do not help management to secure adequate staffing without extra cost (i.e., overstaffing) and results in unfairness regarding unbalanced staff workloads.

Variable shift-length and flexible start-times are useful and practical policies that will ensure an improvement in the quality of schedules regarding coverage, operational efficiency, responsiveness, and flexibility.Moving forward, staff working fixed long shifts are more likely to experience burnout, and job dissatisfaction than staff is working short shifts. However, long shifts are frequent and accepted in hospital systems. Shorter shifts can be incentivized by highlighting the problems of long shifts to encourage employees to sign up for shorter shifts. Allowing staff to work shorter shifts will not only help employees to have better work-life balance but will also help management to retain their staff. To reduce resistance from staff and unions, hospitals should not force their staff to work short shifts. It is recommended to allow staff to sign up for their shift lengths as part of their preferences.

\section{REFERENCES}

Abernathy, W. J., N. Baloff and J.C. Hershey. 1973. "A Three-Stage Manpower Planning And Scheduling Model--A Service-Sector Example”. Operations Research, 21(3), 693-711. 
Aiken, L. H., D. M. Sloane, L. Bruyneel, K. Van Den Heede and W.Sermeus. 2013. "Nurses' Reports Of Working Conditions And Hospital Quality Of Care In 12 Countries In Europe". International Journal Of Nursing Studies, 50(2), 143-153.

Arisha, A. and W. Rashwan. 2016. "Modeling Of Healthcare Systems: Past, Current And Future Trends". In Proceedings Of The 2016 Winter Simulation Conference, edited By T. M. K. Roeder et al. 15231534, Piscataway, New Jersey: IEEE.

Bae, S. H. And D. Fabry. 2013. "Assessing The Relationships Between Nurse Work Hours/Overtime And Nurse And Patient Outcomes: Systematic Literature Review”. Nursing Outlook, 62(2), 138-156.

Brusco, M. J., and L. W. Jacobs. 1995. "Cost Analysis Of Alternative Formulations For Personnel Scheduling In Continuously Operating Organizations”. European Journal Of Operational Research, 86(2), 249-261.

Buchan, J., M. Kingma and F. M. Lorenzo. 2005. "International Migration Of Nurses: Trends And Policy Implications". Bulletin Of The World Health Organization, 82, 587-594.

Buchan, J., and I. Seccombe. 1995. "Managing Nurse". Health Manpower Management, 21(2), 3-12.

Campbell, G. M. 1999. "Cross-Utilization Of Workers Whose Capabilities Differ". Management Science, 45(5), 722-732.

Clark, A., P. Moule, A. Topping and M. Serpell. 2015. "Rescheduling Nursing Shifts: Scoping The Challenge And Examining The Potential Of Mathematical Model Based Tools. Journal Of Nursing Management, 23(4), 411-420.

Costa, N. R., J. Louren and Z. L. Pereira. 2011. "Desirability Function Approach: A Review And Performance Evaluation In Adverse Conditions". Chemometrics And Intelligent Laboratory Systems, 107(2), 234-244.

Dunn, S., B. Wilson and A. Esterman, 2005. "Perceptions Of Working As A Nurse In An Acute Care Setting". Journal Of Nursing Management, 13, 22-31.

Ernst, A., H. Jiang, M. Krishnamoorthy, and D. Sier. 2004. "Staff Scheduling And Rostering: A Review Of Applications, Methods, And Models". European Journal Of Operational Research, 153(1), 3-27.

Hayes, B., and A. Bonnet. 2010. "Job Satisfaction, Stress, And Burnout Associated With Haemodialysis Nursing: A Review Of Literature". Journal Of Renal Care, 36, 174-179.

Hegney, D., A. Plank, and V. Parker. 2006. "Extrinsic And Intrinsic Work Values: Their Impact On Job Satisfaction In Nursing". Journal Of Nursing Management, 14, 271-281.

HRSA. 2002. "Projected Supply, Demand, And Shortages Of Registered Nurses: 2000-2020". Bureau Of Health Professions.

Goodin, J. H. 2003. "The Nursing Shortage In The United States Of America: An Integrative Review Of The Literature". Journal Of Advanced Nursing, 43(4), 335-43.

Jones, C. B. 2005. "The Costs Of Nurse Turnover, Part 2: Application Of The Nursing Turnover Cost Calculation Methodology”. The Journal Of Nursing Administration, 35, 41-49.

Kane, R. L., T. A. Shamliyan, C. Mueller, S. Duval, and T. J. Wilt. 2007. "The Association Of Registered Nurse Staffing Levels And Patient Outcomes”. Medical Care, 45(12), 1195-1204.

Kim, K., and S. Mehrotra. 2015. "A Two-Stage Stochastic Integer Programming Approach To Integrated Staffing And Scheduling With Application To Nurse Management". Operations Research, 63(6), 14311451

Li, Y. J., J. Chen, and X.Q. Cai. 2007. “An Integrated Staff-Sizing Approach Considering Feasibility Of Scheduling Decision". Annals Of Operations Research, 155(1), 361-390.

Morris, J. G., and M. J. Showalter. 1983. "Simple Approaches To Shift, Days-Off And Tour Scheduling Problems". Management Science, 29(8), 942-950.

Murray, M. K. 2002. The Nursing Shortage. Past, Present, And Future. The Journal Of Nursing Administration, 32(2), 79-84.

Norrish, B. R., and T. G. Rundall. 2001. "Hospital Restructuring And The Work Of Registered Nurses". The Milbank Quarterly, 79(1), 55-79. 
Portoghese, I., M. Galletta, R. C. Coppola, G. Finco, and M. Campagna. 2014. "Burnout And Workload Among Health Care Workers: The Moderating Role Of Job Control”. Safety And Health At Work, 5(3), $152-157$.

Rashwan, W. 2017. "An Integrated Framework For Staffing And Shift Scheduling In Hospitals”. DIT.

Rogers, A. E. 2008. "The Effects Of Fatigue And Sleepiness On Nurse Performance And Patient Safety". In Patient Safety And Quality: An Evidence-Based Handbook For Nurses, 509-545.

Rothberg, M. B., I. Abraham, P. K. Lindenauer, and D. N. Rose. 2005. "Improving Nurse-To-Patient Staffing Ratios As A Cost-Effective Safety Intervention”. Medical Care, 43, 785-791.

Silvestro, R., And C. Silvestro. 2008. Towards A Model Of Strategic Roster Planning And Control: An Empirical Study Of Nurse Rostering Practices In The UK National Health Service. Health Services Management Research: An Official Journal Of The Association Of University Programs In Health Administration / HSMC, AUPHA, 21, 93-105.

Sinreich, D., O. Jabali, and N. P. Dellaert. 2012. Reducing Emergency Department Waiting Times By Adjusting Work Shifts Considering Patient Visits To Multiple Care Providers. IIE Transactions, 44(3), $163-180$.

Smith, C. D. A., C. Fisher, and A. Mercer. 2011. Rediscovering Nursing: A Study Of Overseas Nurses Working In Western Australia. Nursing \& Health Sciences, 13(3), 289-95.

Van Den Bergh, J., J. Beliën, P. De Bruecker, E. Demeulemeester and L. De Boeck. 2013. "Personnel Scheduling: A Literature Review". European Journal Of Operational Research, 226(3), 367-385.

Wilson, J. L. 2002. The Impact Of Shift Patterns On Healthcare Professionals. Journal Of Nursing Management, 10(4), 211-219.

Zurn, P., C. Dolea, and B. Stilwell. 2005. Nurse Retention And Recruitment: Developing A Motivated Workforce. The Global Nursing Review Initiative (WHO).

\section{AUTHOR BIOGRAPHIES}

WAEL RASHWAN is a Data Scientist at CeADAR- Centre for Applied Data Analytics and Machine Intelligence- Dublin, Ireland. He holds a Ph.D. in Operations Research and Optimization from Dublin Institute of Technology, Ireland, and MSc. in Optimization from Cairo University. Wael is an active researcher in developing solutions and theories to support and address decision making in uncertain and complex environments. His particular research interests include Machine Learning, Simulation, Optimization, and Healthcare Innovation and Smart Hospitals. Currently, he is leading the healthcare innovation in CeADAR, passionate about using Machine Learning \& Optimization Technologies for solving problems in healthcare and Business domains. His email address is wael.rashwan@dit.ie.

JOHN W. FOWLER is the Motorola Professor in the Supply Chain Management department at ASU. His research interests include discrete event simulation, deterministic scheduling, and multi-criteria decision making. He has published over 120 journal articles and over 100 conference papers. He was the Program Chair for the 2002 and 2008 Industrial Engineering Research Conferences and the 2008 WSC. He was the founding Editor-in-Chief of IIE Transactions on Healthcare Systems Engineering. He is also an Editor of the Journal of Simulation. He is a Fellow of the Institute of Industrial Engineers (IIE) and has served on the WSC Board of Directors from 2005-2017. His email address is john.fowler@asu.edu.

AMR ARISHA, Ph.D. is the Director of the 3S Group and the Head of International Business Department at College of Business, Dublin Institute of Technology (DIT). He Joined DIT in 2005; he received his Ph.D. in Industrial Engineering from Dublin City University (DCU). Intel-Ireland has sponsored his research from 2000 - 2005. His research interests include analysis and optimization of complex dynamic systems in manufacturing and service industries. He is a member of IIE, IMECH, IEI, ESE, ORS, IEEE and ASME and Chief Examiner at MII. His e-mail address is amr.arisha@dit.ie. 